\title{
Pioneers in Optics: George Gabriel Stokes and William Hyde Wollaston
}

\author{
Michael W. Davidson \\ National High Magnetic Field Laboratory, Florida State University, Tallahassee, FL 32306 \\ davidson@magnet.fsu.edu
}

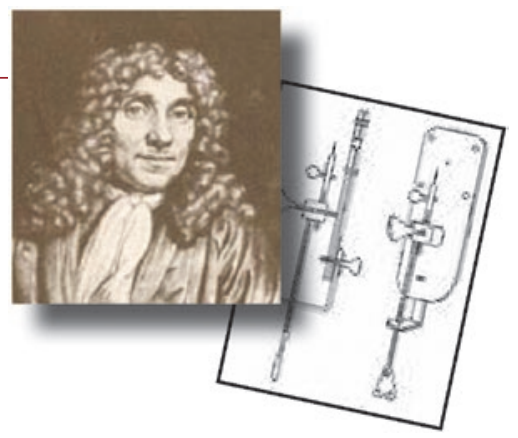

\section{George Gabriel Stokes (1819-1903)}

Born in Ireland on August 13, 1819, George Stokes was the youngest of six children. His father, a rector, directed his early education before sending him to a school in Dublin. Stokes attended Bristol College in England, followed by Pembroke College at Cambridge University, where he studied mathematics. He graduated in 1841 and was bestowed with many honors, including a fellowship that enabled him to remain at Cambridge. In 1849, Stokes was appointed Lucasian Professor of Mathematics and spent the rest of his life working at the prestigious school.

Throughout his career, Stokes emphasized the importance of experimentation and problem solving, rather than focusing solely on pure mathematics. His practical approach served him well, and Stokes made important advances in several fields, most notably hydrodynamics and optics. An advocate of the wave theory of light, Stokes was one of the prominent nineteenth-century scientists who believed in the concept of an ether permeating space, which he supposed was necessary for light waves to travel. Though the existence of an ether was later disproved, Stokes's efforts in the area helped pioneer the science of geodesy, the study of the Earth and its gravitational field.

In hydrodynamics, Stokes investigated a number of phenomena. Working in conjunction with Claude Navier, he developed a series of equations, known as the Navier-Stokes equations, which described the motion of viscous fluids. In 1850, Stokes made another important contribution to the field when he published a treatise in which he mathematically described the fall of an object through a liquid. Based on his equations and the work of others in the field, Stokes was able to explain the formation of clouds in the atmosphere.

An interest in light and its propagation led to Stokes's many experiments in optics. In 1849, he developed instruments and a method for measuring astigmatism in the eye, and by $1851 \mathrm{had}$ invented a device that could be used to analyze polarized light. It was not until 1852, however, that Stokes carried out what was perhaps his most important optical research. The following is an excerpt of his influential findings, which were read to the Royal Society of London on May 27, 1852:

The following researches originated in a consideration of the very remarkable phenomenon discovered by Sir John Herschel in a solution of sulphate of quinine, and described by him in two papers printed in the Philosophical Transactions for 1845, entitled "On a Case of the Superficial Colour presented by a Homogeneous Liquid internally colourless," and "On the Epipolic Dispersion of Light." The solution of quinine, though it appears to be perfectly transparent and colourless, like water, when viewed by transmitted light, exhibits nevertheless in certain aspects, and under certain incidences of the light, a beautiful celestial blue color. It appears from the experiments of Sir John Herschel that the blue colour comes only from a stratum of fluid of small but finite thickness adjacent to the surface by which the light enters. After passing through this stratum, the incident light, though not sensibly enfeebled nor colored, has lost the power of producing the same effect, and therefore may be considered as in some way or other qualitatively different from the original light.

The noteworthy phenomenon observed by Stokes in quinine was that of fluorescence. Though Herschel had noted the occurrence previously, it was Stokes who revealed several important characteristics of the fluorophore. Most importantly, Stokes understood that the celestial blue color was exhibited only near the surface where the light entered the relatively concentrated quinine solution because the ultraviolet radiation of the sun, or other light source, was completely absorbed by only a shallow layer of the substance. As he pointed out, the light was "qualitatively different" after passing beyond this layer, or, more specifically, the ultraviolet was lacking in the remaining radiation. These findings enabled Stokes to formulate what has come to be known as Stokes Law, which holds that the wavelength of emitted fluorescent light is always greater than the wavelength of the exciting light. Closely associated to the law and frequently used for quantification purposes by scientists is Stokes shift, a measurement of the difference in wavelength or frequency between absorbed and emitted quanta.

Stokes's early research in fluorescence served as the basis for much of his future work in optics. For example, he used fluorescence for spectral analysis of ultraviolet radiation and eventually suggested that Fraunhofer lines were electronic transitions of elements that absorbed light of certain wavelengths from the sun's crust. However, during the second half of his career, Stokes expended an increasing amount of time carrying 


\section{Find the truth behind your image.}

Deconvolution

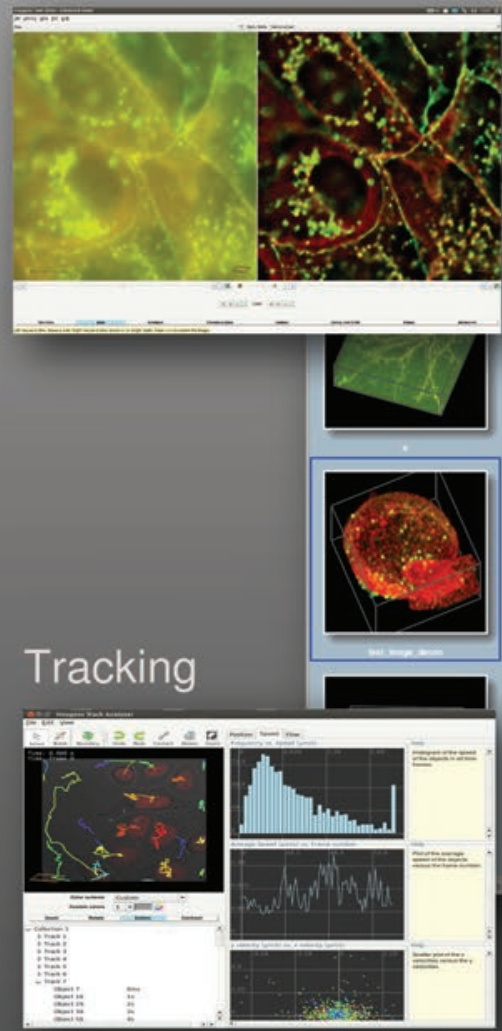

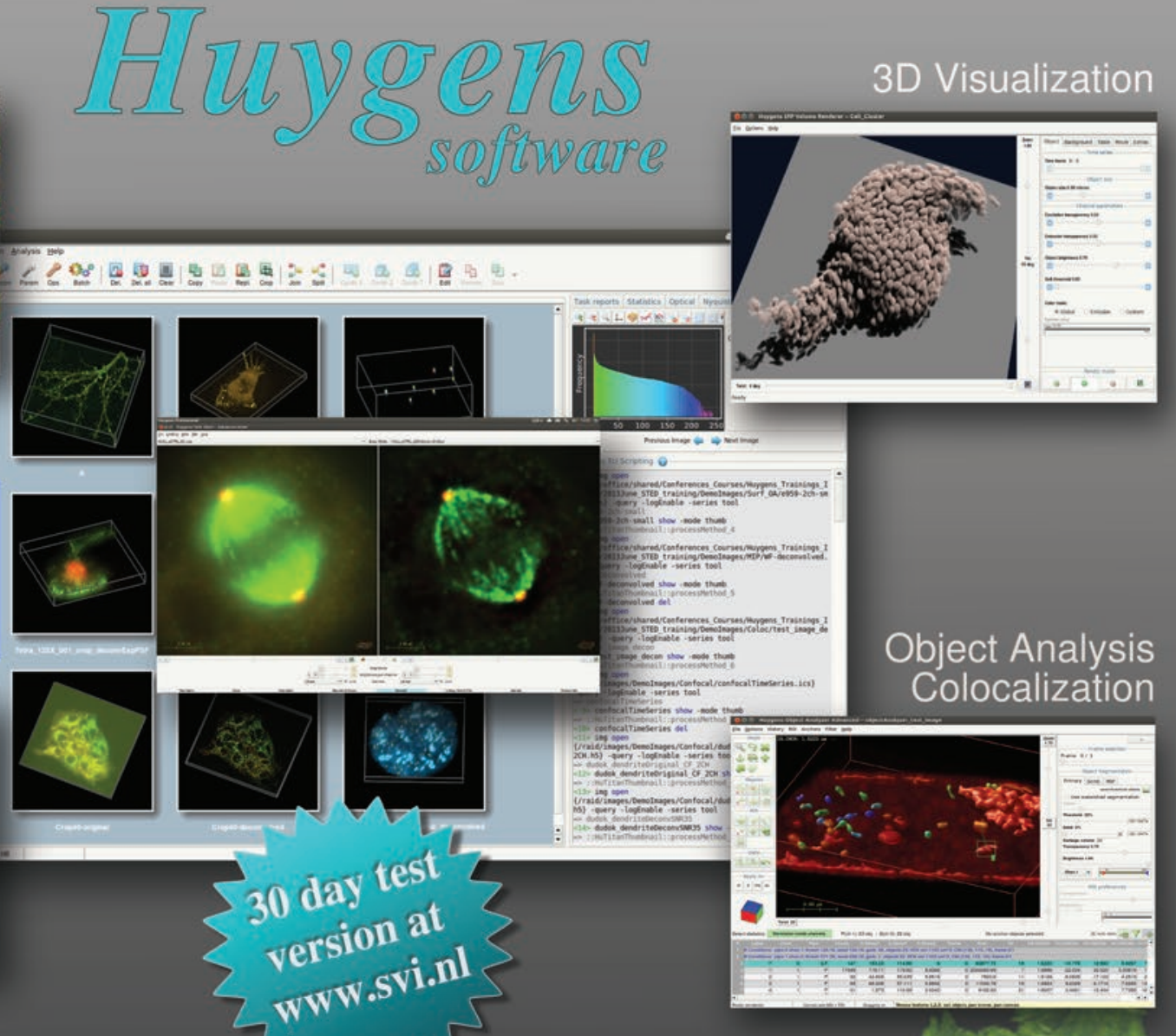

confocal $\bullet$ widefield $\bullet$ STED $\bullet$ spinning disk $\bullet$ multiphoton $\bullet$ brightfield

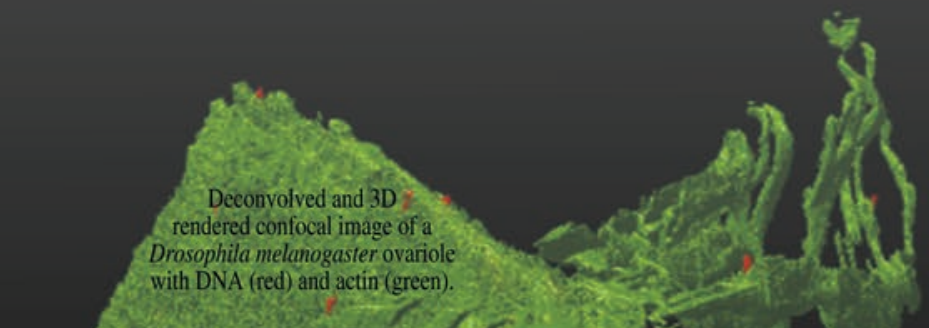


out administrative roles in various academic and political bodies, leaving him with less time to perform original research. Elected to the Royal Society in 1851, Stokes became its secretary in 1854 , and remained in the position until he was elected its president thirty years later. He also served as president of the Cambridge Philosophical Society for six years and acted as a member of Parliament from 1887-1891. For his many scientific contributions, Stokes received a number of honors before his death in 1903, including the Rumford Medal in 1852, the Copley Medal in 1893, and a knighthood in 1889.

\section{William Hyde Wollaston (1766-1828)}

The quantity and diversity of William Hyde Wollaston's research made him one of the most influential scientists of his time. Although formally trained as a physician, Wollaston studied and made advances in many scientific fields, including chemistry, physics, botany, crystallography, optics, astronomy, and mineralogy. He is particularly noted for being the first to observe dark lines in the spectrum of the sun, discovering the elements palladium and rhodium, and proving the elementary nature of niobium and titanium. Wollaston also developed a method for making

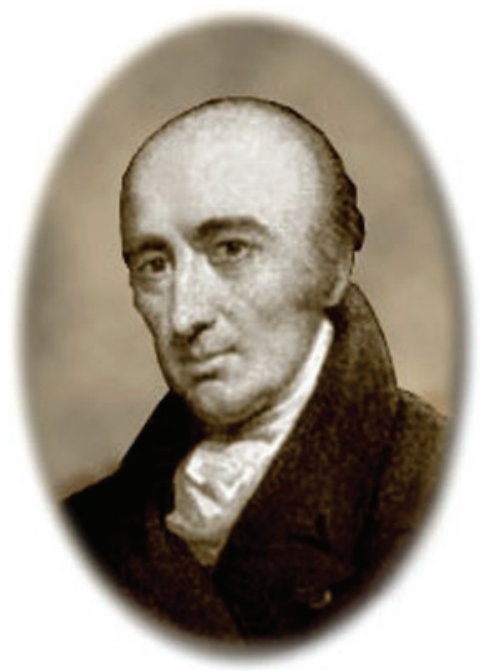
platinum metal malleable, establishing an equivalence between galvanic and frictional electricity, and originating several inventions in optics, including the Wollaston prism that is fundamentally important to interferometry and differential interference contrast (DIC) microscopy.

Wollaston was born in Norfolk, England, on August 6, 1766, to a vicar, Francis Wollaston, and Althea Hyde. He received his formal education at Charterhouse School and the Gonville and Caius Colleges of Cambridge University. He graduated with a degree in medicine and was immediately elected a member of the Royal Society and an associate of the French Academy of Sciences. After practicing as a physician for seven years, he abandoned the field to move to London and focus solely on his research interests. A recipient of the Copley medal in 1802, Wollaston became secretary of the Royal Society in 1806 and served as president from 1820-1828. He lived alone, meticulously conducting his investigations until he died of a brain tumor on December 22, 1828. He donated most of the profits made from his discoveries to scientific societies for further research purposes. The mineral Wollastonite was named in honor of his many achievements.

Wollaston initially became renowned in physiology. In 1797, he described the main components of urinary calculi. Ironically, the future optical pioneer's eyesight soon began failing and, when he suddenly gave up his medical practice in 1800, he became an associate of Humphry Davy at the Royal Institute. While working with Davy in 1809, he described a vibratory action of muscular activity. In 1812, he identified a new type of bladder stone that he named cystic oxide, later called cystine, the first known amino acid. Twelve years later Wollaston provided the best contemporary physiological description of the ear.

Wollaston formed another alliance to perform chemistry studies and experiments, this time with Smithson Tennant. Platinum had long evaded the efforts of chemists to concentrate and purify the precious element, and the pair decided to join in the endeavor. When Tennant first tried to produce platinum, the result was his discovery of the new elements iridium and osmium. Wollaston's later attempt led him to the discovery of palladium and rhodium. He then invented the technique of powder metallurgy and produced malleable platinum in 1805 . The feat proved extremely profitable and provided him with financial independence for the rest of his life. He waited until 1828, however, to reveal the description of his secret process to the Royal Society.

Wollaston made great strides in various disciplines of physics. In 1802, he developed the refractometer, an instrument for determining refractive index. The device helped Wollaston verify the laws of double refraction in Iceland spar, on which he wrote a treatise. Also in 1802, Wollaston discovered that the sun's spectrum is not a continuous gradient but is interrupted by a number of dark lines. Joseph Fraunhofer made the same observation and precisely described the phenomenon twelve years later. In 1807, Wollaston developed a version of the camera lucida, which would eventually lead to Fox Talbot's discoveries in photography.

The camera lucida largely consisted of a four-sided prism (the Wollaston prism) that has since proved indispensable in microscopic work. The invention was originally used as a drawing aid that allowed artists to draw outlines in proper perspective. A piece of paper was laid flat on a drawing board and the artist would look through a lens mounted on a small stand that contained the prism. The observer's eye was placed so that the pupil was half covered by the horizontal face of the prism, allowing him to see both the paper and a faint image of the subject, which he could then easily trace. Although the device was awkward to use, it made artistic renderings significantly simpler to produce and quickly grew in popularity.

As a skilled optician, Wollaston created a doublet lens consisting of two plano-convex lenses with their flat surfaces facing the object plane. This important lens design was refined by Charles Chevalier in 1830 and later perfected by Joseph J. Lister as a high-resolution achromatic lens. In 1829, the doublet lenses, as well as the simple microscope that employed them, were described posthumously. That same year Wollaston's description of a single-lens condenser used to funnel light into a microscope with increased intensity, improving the overall resolution, was also published. Many compound microscopes used Wollaston's system although he did not live to see them, and until recently, microprojectors employed a virtually identical construction.

The Wollaston prism was also instrumental in matching wavefront shears on the surface of prisms. It was improved by Polish physicist Georges Nomarski to what is now known as the Wollaston and Nomarski prism. It is composed of two geometrically identical wedges of quartz or calcite (which are birefringent or doubly refracting materials) cut in a way that their optical axes are oriented perpendicular when they are cemented together to form the prism. If a linear polarizer is oriented so that plane-polarized light enters the prism at a 45-degree angle with respect to the optical axes of the two birefringent prism halves, the light is sheared into two plane-polarized components that are oriented mutually perpendicular to each other. 
Introducing Topspin, a New Digifal STEM, Beam Precession \& Analytical Experiment Framework

TOPSPIN EXPERIMENTS
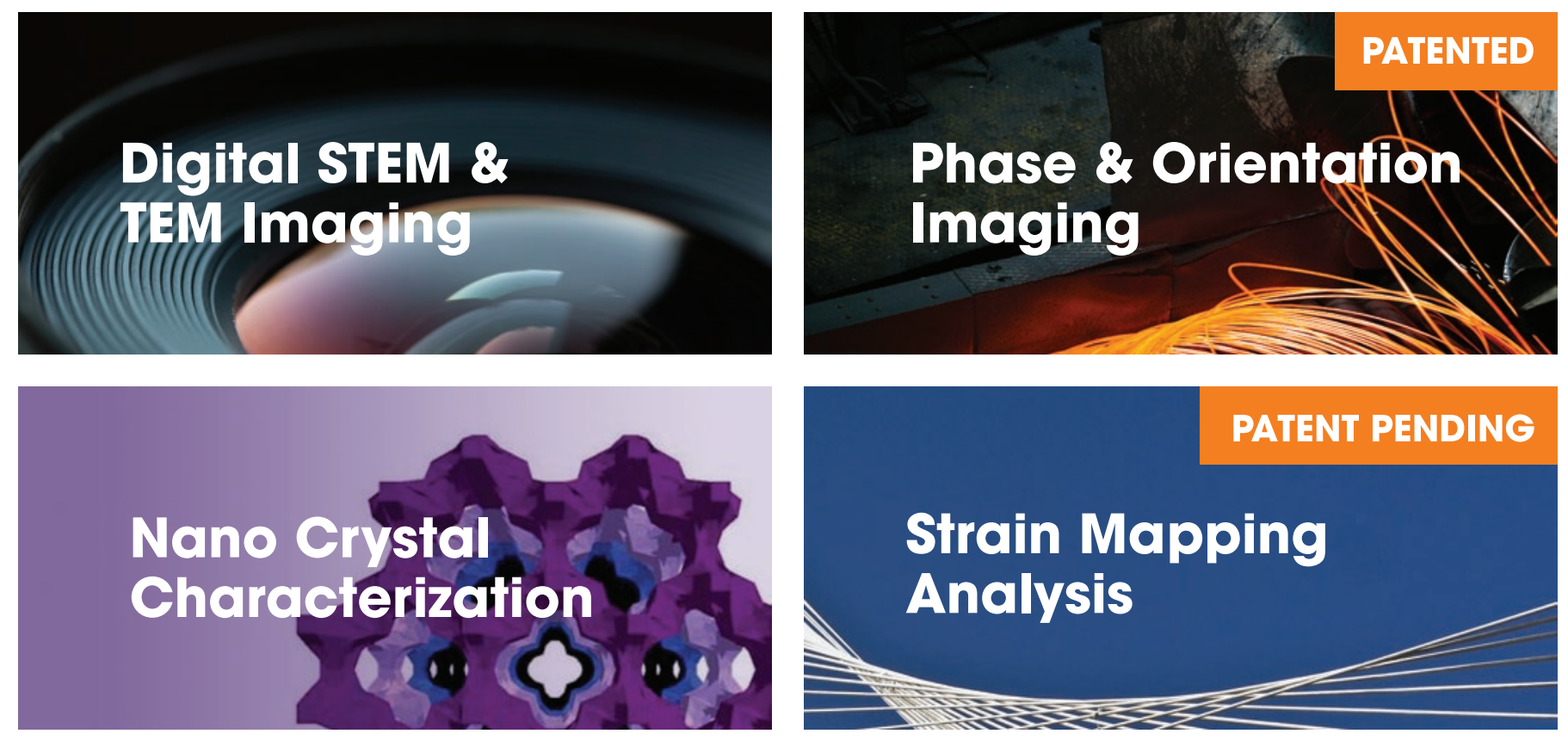

\section{PATENT PENDING}

Enhanced EDX \& EELS Spectroscopy

Model-based EELS Quantification

Have an idea for an experiment? We can help you implement it. 